\title{
The Design of A Novel Sparse Array Using Two Uniform Linear Arrays considering Mutual Coupling
}

\author{
Haiyun Xu $\left(\mathbb{D}\right.$, Weijia Cui $\mathbb{D}^{D}$, Fengtong Mei $\mathbb{D}^{D}$, Bin Ba, and Chunxiao Jian \\ National Digital Switching System Engineering and Technological Research Center, Zhengzhou, Henan Province (450001), China \\ Correspondence should be addressed to Fengtong Mei; 60111mei@sina.com
}

Received 30 March 2021; Revised 17 June 2021; Accepted 21 July 2021; Published 18 August 2021

Academic Editor: He Wen

Copyright (C) 2021 Haiyun Xu et al. This is an open access article distributed under the Creative Commons Attribution License, which permits unrestricted use, distribution, and reproduction in any medium, provided the original work is properly cited.

\begin{abstract}
The sparse arrays using two uniform linear arrays have attracted considerable interest due to the capability of giving analytical expression of sensor location and owning robust direction-of-arrival (DOA) facing strong mutual coupling and sensor failure. In order to achieve the maximum consecutive virtual uniform linear array in difference coarray, in this paper, a design method of a novel sparse array using two uniform linear arrays (NSA-U2) is proposed. We first analyze the relationship between the values of displacement of two subarrays and difference coarray, and then we give the analytical expressions of the displacement and the number of consecutive lags. By discussing the selection of number of subarray sensors, the design of NSA-U2 is completed. Moreover, through choosing a proper compressed interelement spacing, NSA-U2 can be robust to mutual coupling effect. Numerical experiments prove the effectiveness and favorable performance of DOA estimation with mutual coupling.
\end{abstract}

\section{Introduction}

Direction-of-arrival (DOA) estimation is one of the key technologies in the field of passive location. It is widely used in seismic detection, military early warning, astronomical observation, radar monitoring, underwater targets positioning, and so on $[1,2]$. Traditionally, the researchers apply the super resolution methods, such as subspace methods, to solve DOAs mainly in uniform nonsparse arrays, such as uniform linear arrays (ULAs). In recent years, many experts and scholars have focused on nonuniform sparse arrays, whose sensor spacing can be larger than the half of wavelength of impinging signals. Minimum redundancy arrays (MRAs) [3], coprime linear arrays (CLAs) $[4,5]$, and nested linear arrays (NLAs) $[5,6]$ are the typical sparse arrays. Compared with ULAs, the nonuniform sparse arrays can construct the larger array aperture with the same number of sensors, which also means the higher resolution. In order to solve DOAs without any ambiguous values, spatial smoothing multiple signal classification (SS-MUSIC) [7] and direct augmentation approach (DAA) [8] are proposed, respectively. Two algorithms both try to transform the physical array to a virtual nonsparse array, where they both use the difference coarray. [9] demonstrated the effectiveness of two algorithms and concluded that the two share the same performance.

The performance of DOA estimation is mainly decided by the array aperture. While in sparse array, it is changed to be decided by the aperture of consecutive ULA belonging to the difference coarray. Although MRA has the largest virtual ULA aperture in theory, they have no closed-form expression. Thus, a structure named generalized coprime linear array (GCLA) is proposed, where NLA and CLA are the typical arrays. The sensors location of GCLA has an analytical expression, which reduces the complexity of array design. And [10-13] proves that this structure can be much more robust facing sensors failures compared with other sparse arrays. Then, to enlarge the aperture of virtual ULA under the fixed number of sensors, coprime array with compressed interelement spacing (CACIS), coprime array with displaced subarrays (CADiS) [14, 15], coprime array with multiperiod subarrays (CAMpS) [16], and shifted coprime array (SCA) [17] are proposed. CACIS and CADiS show that setting one subarray with a smaller interelement spacing can have the larger aperture of virtual ULA. CAMpS demonstrate that, in order to expand the aperture of virtual ULA, only one 
subarray can have a compressed interelement spacing. SCA reveals that the displacement between two subarrays is the main factor to the aperture of virtual ULA. Based on sparse array using two ULAs, although many valuable researches have been proposed, the solution of how to maximize virtual ULA aperture corresponding to different parameters has not been answered and is still deserved to study.

Moreover, besides the array aperture, the mutual coupling is another factor affecting the performance of DOA estimation. The severe mutual coupling causes the degrade of accuracy. To tackle the problem, the researchers aim to enlarge the interelement spacing and propose super nested arrays (SNAs) [18, 19], the augmented nested array (ANA) [20], and the maximum interelement spacing constraint (MISC) [21]. Based on the GCLA, they divide the dense subarray into three or four sparse subarrays, which decreases the weight of interelement spacing equal to half of wavelength. However, when the mutual coupling is strong, the weight of interelement spacing bigger than half of wavelength should be considered.

Hence, in this paper, based on GCLA configuration, we propose a novel sparse array using two ULAs (NSA-U2). We first derive the analytical expression of displacement between two subarrays, which can decrease the complexity of array design, and then discuss the selection of interelement spacing of each subarray to suppress the strong mutual coupling. Moreover, the effectiveness of conclusion is demonstrated by simulation experiments. The main contribution of this paper is summarized as follows:

(1) Under the fixed number of sensors and same interelement spacing, the paper gives the design of NSA$\mathrm{U} 2$, which can obtain the biggest aperture of the virtual ULA compared with other arrays using two ULAs. Moreover, the paper derives the analytical expression of displacement and the aperture of the consecutive virtual ULA and gives a low-complexity array design method

(2) The paper analyzes the selection of interelement spacing of each subarray, which ensures that the weight of small interelement spacing is low. When mutual coupling is strong, robust DOA estimation can be obtained

The rest of this paper is organized as follows: Section II presents the model of sparse array. Section III proposes the design method of NSA-U2 in detail. Section IV analyzes the performance facing mutual coupling in NSA-U2. Section V is the simulation experiments. Section VI summarizes the paper. Throughout the paper, we make use of the following notations shown in Table 1.

\section{Problem Formulation}

Assume that there are $T$ sensors. Based on GCLA [15], NSA-U2 is made of two ULAs, having $M^{\prime}=M-1$ and $N$ sensors, respectively, where $T=M^{\prime}+N$ and $M$ and $N$ are coprime integers (generally assuming $M<N$ ). We set the
TABLE 1: Key notations used in this paper.

\begin{tabular}{lc}
\hline Transpose & $(\bullet)^{T}$ \\
Conjugate & $(\bullet)^{*}$ \\
Hermitian transpose & $(\bullet)^{H}$ \\
Vectorization operation & $\operatorname{vec}(\bullet)$ \\
Khatri-Rao & $\circ$ \\
Kronecker product & $\otimes$ \\
Diagonal matrix & $\operatorname{diag}[\bullet]$ \\
Floor integer & $\lfloor\bullet]$ \\
Identity matrix with size $M \times M$ & $\mathbf{I}_{M}$ \\
Number of elements & $<\bullet>$ \\
\hline
\end{tabular}

unit interelement spacing to $\lambda / 2$, where $\lambda$ is the wavelength of impinging signals. So, the sensors' location set is all integers, defined as

$$
\mathbb{D}=\{\bar{M} n \mid 0 \leq n \leq N-1\} \cup\left\{\bar{M}(N-1)+L+N m \mid 0 \leq m \leq M^{\prime}-1\right\},
$$

where $\bar{M}=M / p$ and $\bar{M}$ and $p$ are integers. Here, $p$ is the compressed factor, $\bar{M}, N$ are still coprime integers, and $L$ is the displacement between two subarrays. Suppose that there are $K$ far-field narrowband signals impinging on this array from $\left\{\theta_{1}, \cdots, \theta_{K}\right\}$ with power $\left\{\sigma_{1}^{2}, \cdots, \sigma_{K}^{2}\right\}$. Then, the received data is

$$
\mathbf{X}(t)=\sum_{k=1}^{K} \mathbf{a}_{\mathbb{D}}\left(\theta_{k}\right) S_{k}(t)+\mathbf{N}(t)=\mathbf{A}(\theta) \mathbf{S}(t)+\mathbf{N}(t)
$$

where the manifold matrix $\mathbf{A}(\theta)$ is denoted as

$$
\mathbf{A}(\theta)=\left[\mathbf{a}_{\mathbb{D}}\left(\theta_{1}\right), \cdots, \mathbf{a}_{\mathbb{D}}\left(\theta_{K}\right)\right]^{T},
$$

and the steering vector $\mathbf{a}_{\mathbb{D}}(\theta)$ can be given by

$$
\mathbf{a}_{\mathbb{D}}(\theta)=\left[1, e^{-j \pi \ell_{1} \sin \theta}, \cdots, e^{-j \pi \ell_{T-1} \sin \theta}\right]^{T},
$$

where $\ell_{m} \in \mathbb{D}, m=1, \cdots, T-1$. The signal data vector is

$$
\mathbf{S}(t)=\left[S_{1}(t), \cdots, S_{K}(t)\right]^{T},
$$

where $t=1, \cdots, J$, and $J$ is the number of snapshots. And the noise vector is usually Gaussian random variables with zero means and variance $\sigma_{n}^{2}$. From the references mentioned above in Section I, the covariance matrix is use to construct the virtual array. Then, we can have the covariance matrix of the received data denoted as

$$
\mathbf{R}_{X}=\frac{1}{J} \mathbf{X} \mathbf{X}^{H}=\mathbf{A} \mathbf{R}_{S} \mathbf{A}^{H}+\sigma_{n}^{2} \mathbf{I}_{M},
$$


where $\mathbf{R}_{S}=\operatorname{diag}\left[\sigma_{1}^{2}, \cdots, \sigma_{K}^{2}\right]$. Vectorize the covariance matrix $\mathbf{R}_{X}$ and obtain the vector

$$
\mathbf{Z}=\operatorname{vec}\left(\mathbf{R}_{X}\right)=\mathbf{B} \mathbf{p}+\sigma_{n}^{2} \mathbf{e},
$$

where $\mathbf{B}=\mathbf{A}^{*} \circ \mathbf{A}, \mathbf{e}=\operatorname{vec}(\mathbf{I})$, and $\mathbf{p}$ are the diagonal elements of $\mathbf{R}_{S}$. Hence, the vector $\mathbf{Z}$ can be seen as the received data of a virtual linear array $[4,15]$, whose location is defined as the difference coarray, given by

$$
\mathbb{D}_{v}=\mathbb{D}_{s} \cup \mathbb{D}_{s}^{-} \cup \mathbb{D}_{c} \cup \mathbb{D}_{c}^{-},
$$

where the difference coarray is the union of self-difference coarray $\mathbb{D}_{s}$, where

$$
\mathbb{D}_{s}=\left\{\ell_{s} \mid \ell_{s}=\bar{M} n\right\} \cup\left\{\ell_{s} \mid \ell_{s}=N m\right\},
$$

crossdifference coarray $\mathbb{D}_{c}$, where

$$
\mathbb{D}_{c}=\left\{\ell_{c} \mid \ell_{c}=\bar{M}(N-1)+L+N m-\bar{M} n\right\},
$$

and the corresponding mirrored coarray $\mathbb{D}_{s}^{-}=\left\{-\ell_{s} \mid \ell_{s} \in \mathbb{D}_{s}\right\}$ and $\mathbb{D}_{c}^{-}=\left\{-\ell_{c} \mid \ell_{c} \in \mathbb{D}_{c}\right\}$. And the aperture of virtual ULAs in coarray is equivalent to the maximum number of consecutive lags in $\mathbb{D}_{v}$.

CACIS and CADiS $[14,15]$, respectively, set $L=-\bar{M}(N$ -1 ) and $L=\bar{M}+N$. Comparing CACIS with CADiS, we notice that CACIS has the bigger consecutive integer range while it can still be improved, and CADiS has the bigger number of unique coarray lags while its consecutive integer range is much smaller. Hence, the value of $L$ is a main factor to the number of consecutive lags. SCA in [17] gives a solution that when $p=1$, setting $L=\lfloor M / 2\rfloor N-\bar{M}(N-1)$ can obtain the maximum number of consecutive lags. Then, we need to find a more general solution of $L$ under different $p$. Because $p=M$ is a special case, where nested CADiS in [?] reveals that $L=N+1$ can have the maximum number of consecutive lags, we will take the $1 \leq p<M$ into consideration in the following section.

\section{Proposed Array Design Method}

In order to have the maximum number of consecutive lags, defined as $S_{v}$, the key of the NSA-U2 design method is to solve the value of $L$ and the selection of $M, N$ with the fixed $T$. In this section, we will, respectively, give the setting of $L$ and $M, N$ with the detailed proof.

3.1. The Analytical Expression of L. Based on (8), we solve the analytical expression of $L$ in two steps. The first step is solving $L$ to obtain the maximum number of consecutive lags in $\mathbb{D}_{c} \cup \mathbb{D}_{c}^{-}$. The second step is adjusting $L$ when considering the effect of $\mathbb{D}_{s} \cup \mathbb{D}_{s}^{-}$to $\mathbb{D}_{v}$.

In first step, in order to obtain the maximum number of consecutive lags of $\mathbb{D}_{c} \cup \mathbb{D}_{c}^{-}$, the positive and negative lags must be connected. Before solving $L$, we introduce the proposition proposed in [15], which tells the analytical expression of lags and holes in $\mathbb{D}_{c}$ :

\section{Proposition 1.}

$\mathbb{D}_{c}$ contains the continuous integer $\ell_{c}$, where $(\bar{M}-1)(N$ $-1)+L \leq \ell_{c} \leq M^{\prime} N-1+L$

$\mathbb{D}_{c}$ contains the holes located at $\bar{M}(N-1)-(p \bar{M}+q N)$ $+L$, where $p \geq 0, q>0$ are integers

Then, we further propose the proposition 2 based on Proposition 1:

Proposition 2. $\mathbb{D}_{c}$ meets the following properties:

(a) The lags in $\mathbb{D}_{c}$ can be divided into three parts, which are given by $\mathbb{D}_{c n}=\left\{\ell_{c} \mid \ell_{c}=L+a \bar{M}+b N, \ell_{c}<(\bar{M}-1\right.$ )$(N-1)+L, a \geq 0, b \geq 0\}, \mathbb{D}_{c c}=\left\{\ell_{c} \mid(\bar{M}-1)(N-1)\right.$ $\left.+L \leq \ell_{c} \leq M^{\prime} N-1+L\right\}$, and $\mathbb{D}_{c p}=\left\{\ell_{c} \mid \ell_{c}=\bar{M}(N-\right.$ 1) $+L+\left(M^{\prime}-1\right) N-a \bar{M}-b N, \ell_{c}>M^{\prime} N-1+L, a$ $\geq 0, b \geq 0\}$

(b) The holes in $\mathbb{D}_{c}$ can be divided into two parts, which are given by $\mathbb{D}_{h n}=\left\{\ell_{h} \mid \ell_{h}=(\bar{M}-1)(N-1)+L-1\right.$ $\left.-(a \bar{M}+b N), \ell_{h}>L, a \geq 0, b \geq 0\right\}$ and $\mathbb{D}_{h p}=\left\{\ell_{h} \mid \ell_{h}\right.$ $=M^{\prime} N+L+(a \bar{M}+b N), \ell_{h} \leq \bar{M}(N-1)+L+\left(M^{\prime}\right.$ $-1) N, a \geq 0, b \geq 0\}$

Proof.

(a) Due to the property (a) of Proposition 1, we can have that $\mathbb{D}_{c c}$ contains all consecutive integers of $\mathbb{D}_{c} . \mathbb{D}_{c n}$ contains the values, which are not consecutive and less than $(\bar{M}-1)(N-1)+L$. The minimum value of $\mathbb{D}_{c n}$ is $L$, where $m=0, n=N-1$. The value no less than $L$ can be denoted by $N(0+b)-\bar{M}(N-1-a)$, where $a \geq 0, b \geq 0$. So, any value in $\mathbb{D}_{c n}$ is given by $\ell_{c}$ $=L+a \bar{M}+b N . \mathbb{D}_{c p}$ contains the values, which are not consecutive and more than $M^{\prime} N-1+L$. Similarly, The maximum value of $\mathbb{D}_{c p}$ is $\bar{M}(N-1)+L+$ $\left(M^{\prime}-1\right) N$, where $m=M^{\prime}-1, n=0$. The value no more than it can be denoted by $N(M-2-b)-\bar{M}(0$ $+a)$, where $a \geq 0, b \geq 0$. So, any value in $\mathbb{D}_{c p}$ is given by $\ell_{c}=\bar{M}(N-1)+L+\left(M^{\prime}-1\right) N-a \bar{M}-b N$

(b) The holes can be divided into two parts, where $\mathbb{D}_{h n}$ corresponds to the holes in $\mathbb{D}_{c n}$, and $\mathbb{D}_{h p}$ corresponds to the holes in $\mathbb{D}_{c p}$. The maximum value of $\mathbb{D}_{h n}$ is ( $\bar{M}-1)(N-1)+L-1$. Considering the property (b) of Proposition 1 , any hole no more than $(\bar{M}-1)(N$ $-1)+L-1$ is given by $\ell_{h}=(\bar{M}-1)(N-1)+L-1$ $-(a \bar{M}+b N)$, where $a \geq 0, b \geq 0$. Similarly, the minimum value of $\mathbb{D}_{h p}$ is $M^{\prime} N+L$. So, any hole no less than $M^{\prime} N+L$ is given by $\ell_{h}=M^{\prime} N+L+(a \bar{M}+b N$ ), where $a \geq 0, b \geq 0$

The maximum number of consecutive lags of $\mathbb{D}_{c} \cup \mathbb{D}_{c}^{-}$ means that positive and negative lags are connected. Thus, 
TABLE 2: The analytical expressions of $L$ and $S_{v}$.

\begin{tabular}{lcc}
\hline Parameters & $L$ & $S_{v}$ \\
\hline$p=1$, & $-(\bar{M} N-\bar{M}) / 2$ & $M N+M+2 N-1$ \\
$\bar{M}$ is even, and $N$ is odd & $-(\bar{M} N-N) / 2$ & $M N+2 N-1$ \\
$p=1, N>2 \bar{M}$, & $-\bar{M} N / 2$ & $M N+N+2 M-1$ \\
$\bar{M}$ is odd, and $N$ is even & & $M N+M+2 N-1$ \\
$p=1, N \leq 2 \bar{M}$, & $-(\bar{M} N-\bar{M}) / 2$ & $M$ \\
$\bar{M}$ is odd, and $N$ is even & $-\bar{M} N / 2$ & $2 M N-\bar{M} N+2 \bar{M}-1$ \\
$p=1$, & $-(\bar{M} N-N) / 2$ & $2 M N-\bar{M} N+N+2 \bar{M}-1$ \\
$\bar{M}$ is odd, and $N$ is odd & & \\
$p>1$, & $-(\bar{M} N-N) / 2$ & $2 M N-\bar{M} N+N+2 \bar{M}-1$ \\
$\bar{M}$ is even, and $N$ is odd & \\
$p>1$, & \\
$\bar{M}$ is odd, and $N$ is even & \\
$p>1$, & & \\
$\bar{M}$ is odd, and $N$ is odd & & \\
\hline
\end{tabular}

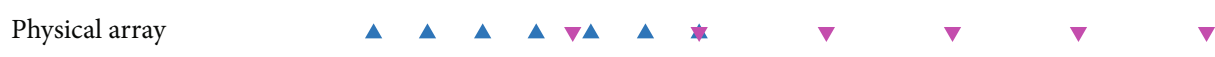

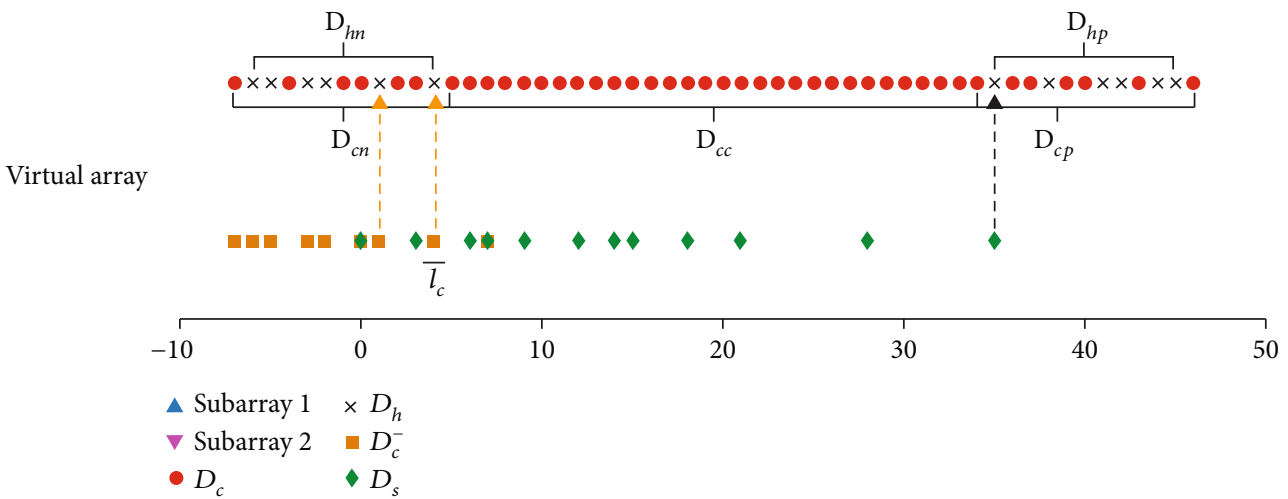

Figure 1: An example when $M=6, N=7$, and $p=2$.

we should let the number of holes in $\mathbb{D}_{c n}$ be aligned by $\mathbb{D}_{c n}^{-}$as many as possible. Through the analysis in Appendix A, we give the theorem about the expression of $L$ :

Theorem 3. $\mathbb{D}_{c} \cup \mathbb{D}_{c}^{-}$can achieve the maximum number of consecutive lags defined as $S_{c}=2(M N+L)-1$, when

$$
L=-\frac{1}{2}\left(\bar{M} N-\bar{M}-N+a_{1} \bar{M}+a_{2} N\right)
$$

where the integers $a_{1}, a_{2} \in\{0,1\}$ and $a_{1}, a_{2}$ cannot equal to 0 simultaneously. Moreover, the values of $a_{1}, a_{2}$ are depended on the odevity of $\bar{M}, N$.

Proof. See Appendix A.

In second step, with the theorem 3 , we use $\mathbb{D}_{s} \cup \mathbb{D}_{s}^{-}$to fill the holes in $\mathbb{D}_{c p} \cup \mathbb{D}_{c p}^{-}$and obtain the detailed expression of $L$, which is the function of $\bar{M}, N$. We propose the theorem 4 , which states that
TABLE 3: The values of $L, m$, and $n$ meeting equation (A.3).

\begin{tabular}{lccc}
\hline Parameters & $L$ & $m$ & $n$ \\
\hline $\bar{M}$ is odd & $-\frac{\bar{M} N-N}{2}$ & $\frac{\bar{M}-1}{2}$ & $N-1$ \\
$N$ is even/odd & $\bar{M} N$ & $\bar{M}$ & $N-1$ \\
$\bar{M}$ is even & $-\frac{\overline{2}}{2}$ & & $N-1$ \\
$N$ is even/odd & $\bar{M} N-\bar{M}$ & 0 & $\frac{N}{2}$ \\
$\bar{M}$ is even/odd & $-\frac{\bar{N}}{N \text { is odd }}$ & &
\end{tabular}

Theorem 4. The analytical expression of $L$ and $S_{v}$ are showed in Table 2.

Proof. See Appendix B.

We show an example in Figure 1 , where $M=6, N=7$, and $p=2$. Thus, $L=-(\bar{M} N-N) / 2=-7$ from Table 2 . The physical sensors of two subarrays have one common sensor located at the $N$ th sensor of subarray 1 and $(\bar{M} / 2+1 / 2)$ th sensor of subarray 2 , which proves the Table 3 . And $\ell_{c}=\bar{M}$ $N-\bar{M}-N+L=4$; so, positive holes in $\mathbb{D}_{c}$ can be aligned 

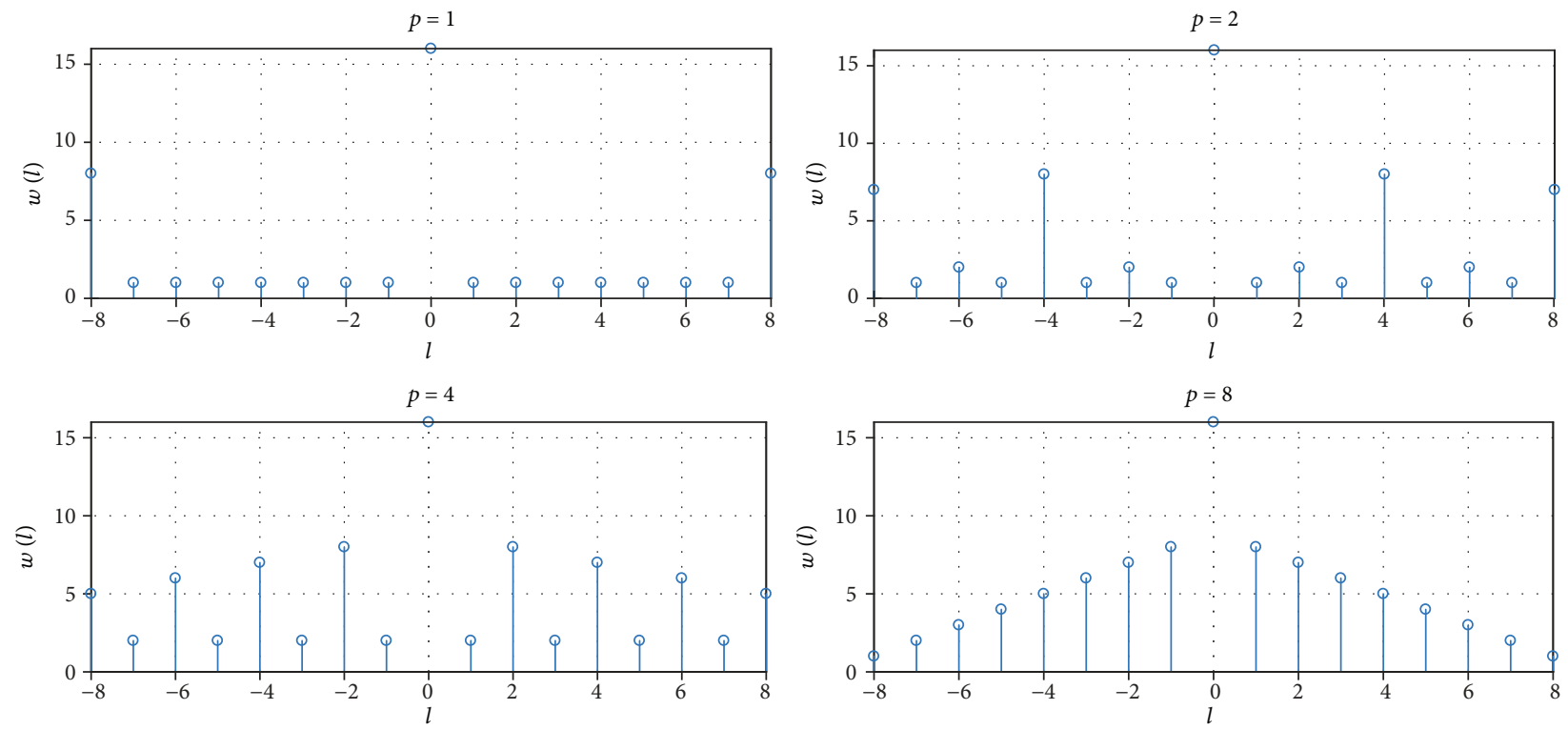

Figure 2: The weight function of $\mathbb{D}_{v}$ under different $p$ with $T=16$.

by $\mathbb{D}_{c}^{-}$, which proves the Theorem 3 , and the minimum value in $\mathbb{D}_{h p}$ can be aligned by $\mathbb{D}_{s}$, which proves the Theorem 4 . Hence, the figure demonstrates that the proposed theorems are right.

3.2. The Selection of Sensor Number of Each Subarray. From Table 2, we have the analytical expression of $L$ and $S_{v}$, which is determined by $\bar{M}$ and $N$. With a fixed number of sensors $T$, the values of $(\bar{M}, N)$ meet the following theorem to maximize the value of $S_{v}$.

Theorem 5. With the fixed values of $T$ and $p$, when $(M, N)$ satisfies

$$
\left\{\begin{array}{ll}
M=\frac{T}{2}, N=\frac{T}{2}+1 & T \text { is even } \\
M=\frac{T-1}{2}, N=\frac{T+3}{2} & T \text { is odd }
\end{array},\right.
$$

the value of $S_{v}$ achieves the maximum. Moreover, under the selection of $(M, N)$, with the increase of $p, S_{v}$ increases.

Proof. See Appendix C.

In conclusion, with a fixed $T$, we take the values of $\bar{M}$, $N, L$ into (1) and can have the sensors location of NSA-U2.

\section{The Proposed Sparse Array Facing Mutual Coupling}

4.1. The Mutual Coupling and DOA Estimation. When there exists mutual coupling between physical sensors, (2) can be changed to

$$
\boldsymbol{X}(t)=\boldsymbol{C A}(\theta) \boldsymbol{S}(t)+\boldsymbol{N}(t)
$$

where $\mathbf{C}$ is the mutual coupling matrix with size $T \times T$. Generally, in linear array, $\mathbf{C}$ can be a $B$-band symmetric Toeplitz matrix [22-27]. The element in $m$ th row and $n$th column is defined as

$$
[\mathbf{C}]_{m, n}= \begin{cases}c_{\left|\ell_{m}-\ell_{n}\right|}, & \left|\ell_{m}-\ell_{n}\right|<B \\ 0, & \text { otherwise, }\end{cases}
$$

where $c_{0}, c_{1}, \cdots, c_{B}$ are coupling coefficients satisfying $c_{0}=1$ $>c_{1}>\cdots>c_{B}$. (14) reveals that the sparse array that has a much larger interelement spacing can have weaker mutual coupling. To quantify the effect of mutual coupling, we introduce the following definitions [18-21]:

Definition 6. The weight function of the virtual array $\mathbb{D}_{v}$ is defined as the number of coarray lags index $\ell$, which can be expressed as

$$
w(\ell)=<\left\{\left(\ell_{m}, \ell_{n}\right) \mid \ell_{m}-\ell_{n}=\ell\right\}>, \ell_{m}, \ell_{n} \in \mathbb{D}
$$

When $|\ell|<B$, the value of $w(\ell)$ is smaller, and the mutual coupling is less significant.

Definition 7. The coupling leakage is defined as

$$
L e=\frac{\|\mathbf{C}-\operatorname{diag}(\mathbf{C})\|_{F}}{\|\mathbf{C}\|_{F}},
$$

where the smaller value of Le implies the weaker mutual coupling. 


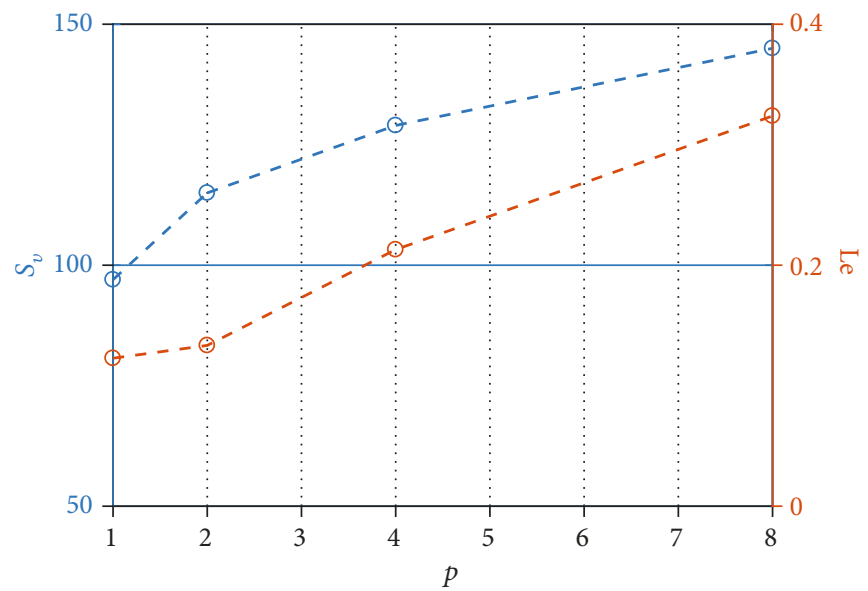

(a)

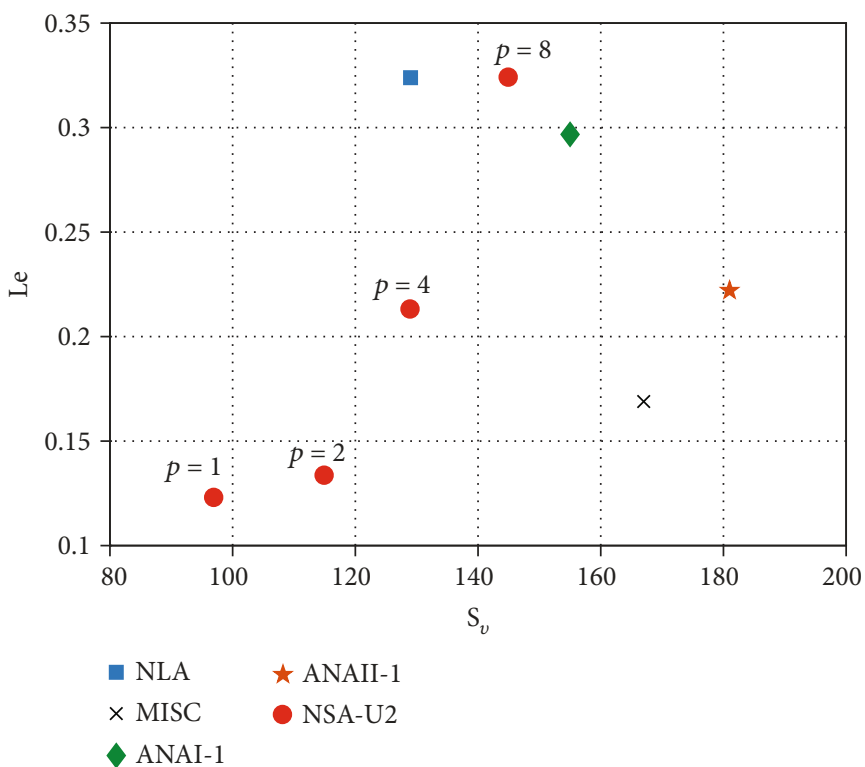

(b)

FIgURE 3: The relationship between $S_{v}$ and $L e$, (a) under different $p$ and (b) under different sparse arrays.

Thus, we can have the corresponding proposition about the mutual coupling of NSA-U2:

Proposition 8. The weight function of $\mathbb{D}_{v}$ is as follows:

(a) The weight function of $\mathbb{D}_{c}$ has $w(\ell)=1$

(b) The weight function of $\mathbb{D}_{c} \cup \mathbb{D}_{c}^{-}$has

$$
w(\ell)= \begin{cases}2 & \ell=0 \\ 2 & \ell= \pm\left(\bar{\ell}_{c}+a \bar{M}+b N\right),|\ell| \leq-L \\ 1 & \text { otherwise }\end{cases}
$$

where $a \geq 0, b \geq 0$ and $a, b$ cannot be 0 at the same time.

(c) The weight function of $\mathbb{D}_{s} \cup \mathbb{D}_{s}^{-}$has
$w(0)=T, w( \pm \bar{M})=N-1, w( \pm N)=M-1$.

(d) The weight function of $\mathbb{D}_{v}$ is the sum of that of $\mathbb{D}_{c} \cup \mathbb{D}_{c}^{-}$ and $\mathbb{D}_{s} \cup \mathbb{D}_{s}^{-}$

Proof. See Appendix D.

Due to Proposition 8 , in order to have the weak mutual coupling, the value of $w(\ell)(\ell<B)$ should be small. So, the value of $p$, which decides the value of $\bar{M}$, is important to the values of $w(\ell)$ and $L$. The detailed analysis will show in the next part.

Applying DAA to the received data (7) of virtual sensors, we can have the MUSIC spectrum and obtain the DOA of signals. 

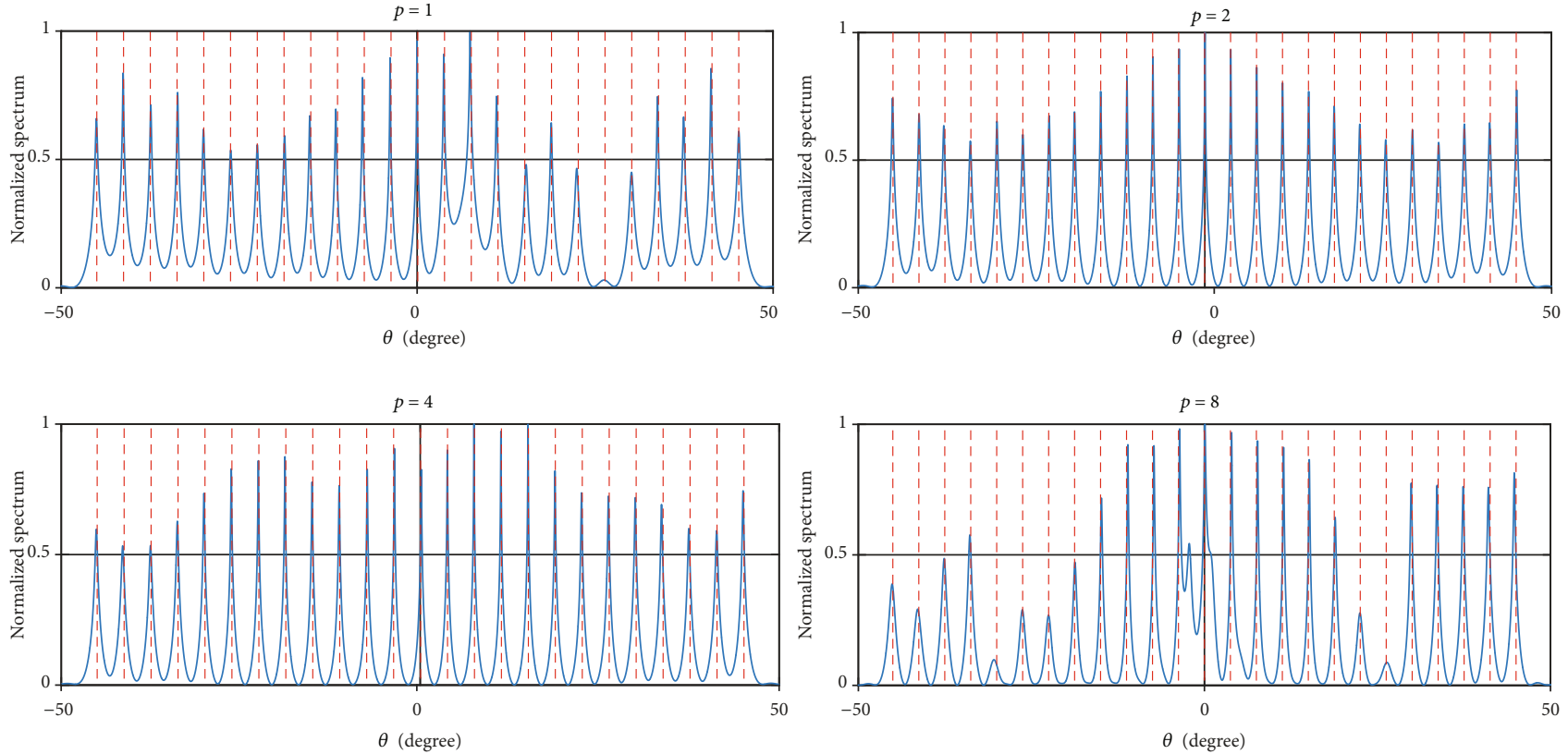

FIgURE 4: The MUSIC spectrum under different $p$.
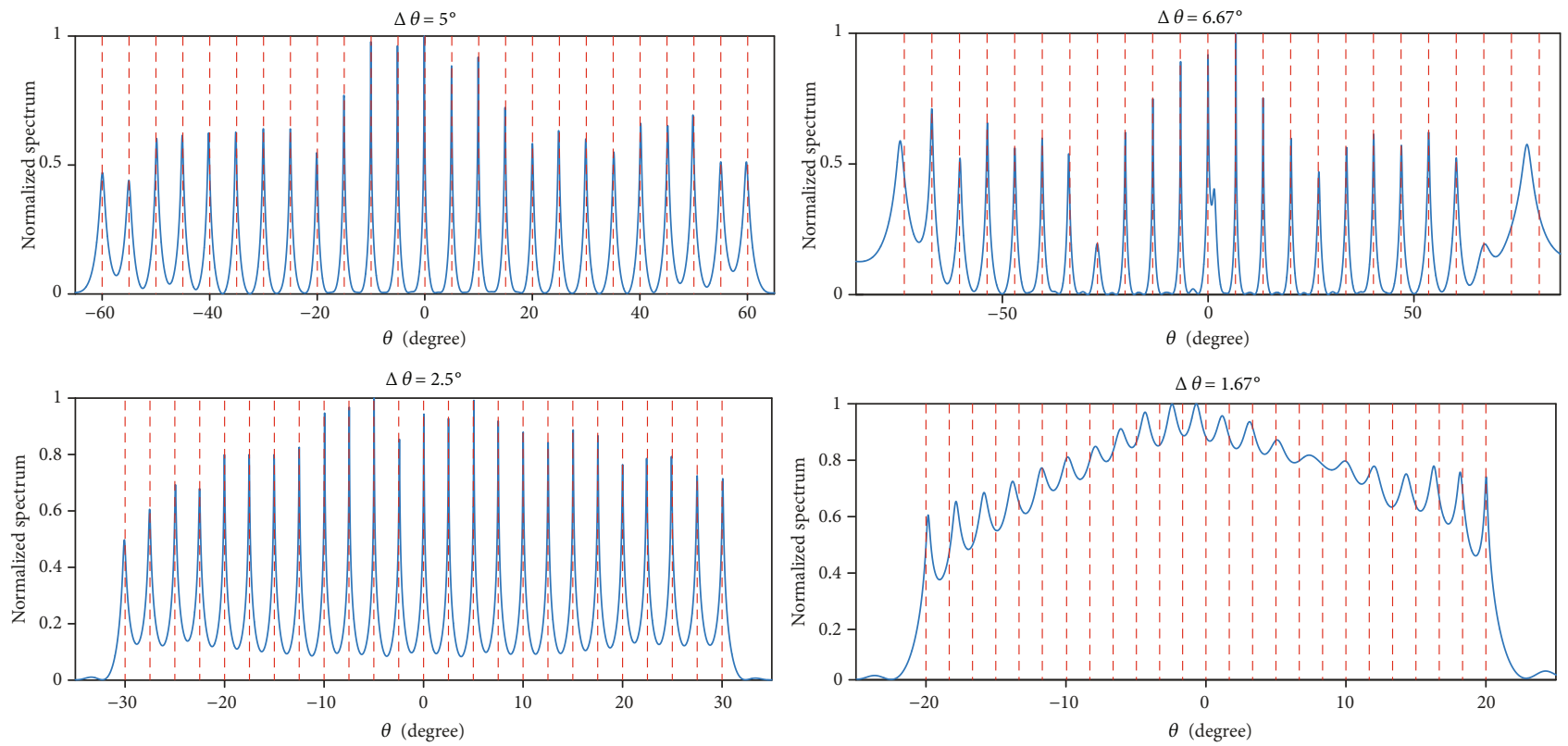

FIgURE 5: The MUSIC spectrum under different $\Delta \theta$.

4.2. The Relationship between $p$ and Le. With a fixed value of $T$, although a big $p$ can have a big $S_{v}$, a small $\bar{M}$ can cause a big $L e$. In order to show the relationship, we set $T=16$, and based on Proposition $8, M=8, N=9$. Thus, $p$ can be $\{1,2$, $4,8\}$. Firstly, we resolve the weight function of difference coarray $\mathbb{D}_{v}$ corresponding to different values of $p$, which is shown in Figure 2. The figure presents that if $p$ is small, when $\ell$ is small, $w(\ell)$ can be 1 . In order to quantify the influence of mutual coupling, we assume that $B=3, c_{1}=0.3 e^{j \pi / 3}$, and $c_{b}=c_{1} e^{-j \pi(b-1) / 8}$. Moreover, we compare NSA-U2 with the existing sparse arrays, such as NLA [6], MISC [20], ANAI-1, and ANAII-1 [20], under the same number of sen- sors. Figure 3(a) shows the relationship between $S_{v}$, Le and $p$ in NSA-U2. With the increase of $p, S_{v}$ increases, but Le also increases. When mutual coupling is severe, a big array aperture may not obtain the high accuracy. Figure 3(b) compares $S_{v}$, Le of different kinds of sparse arrays with same $T$ - When $p=1$ and $p=2$, NSA-U2 can have a small Le but also a small $S_{v}$. ANAII-1 and MISC have the larger $S_{v}$ and also the bigger Le.

In conclusion, the selection of $p$ needs to balance the values of $S_{v}$ and Le to obtain a favorable performance, where the influence to DOA estimation will be presented by simulation experiments. 


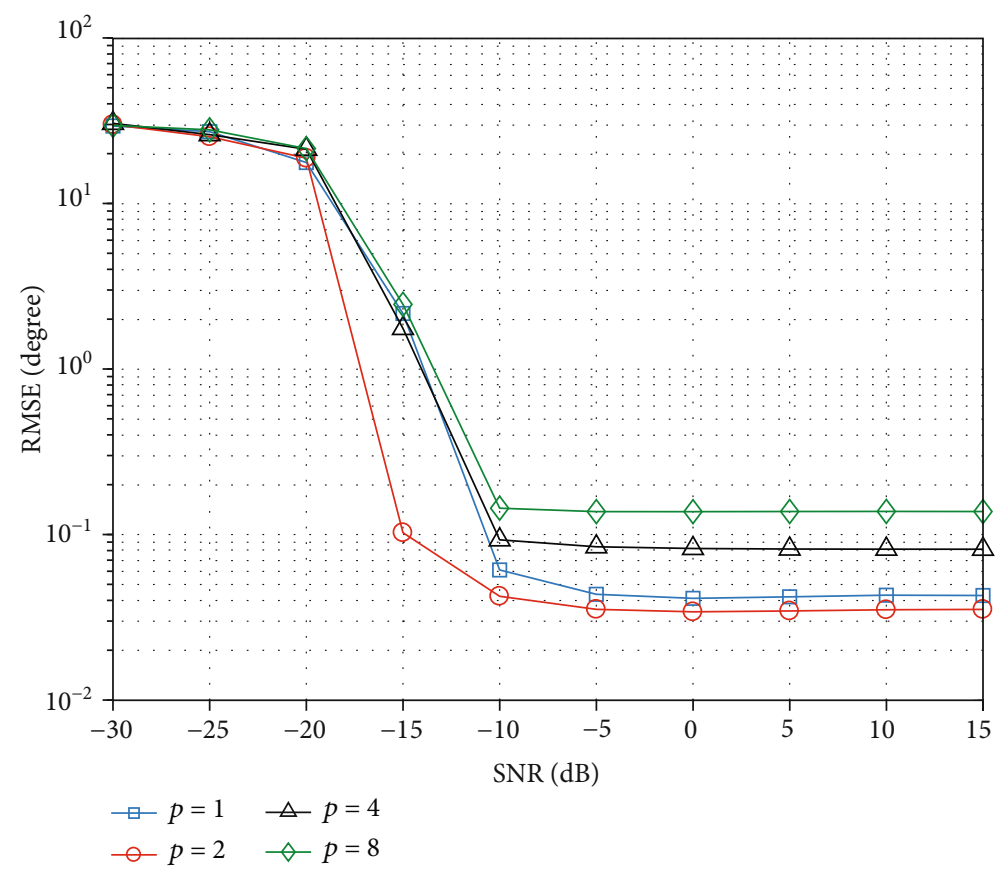

(a)

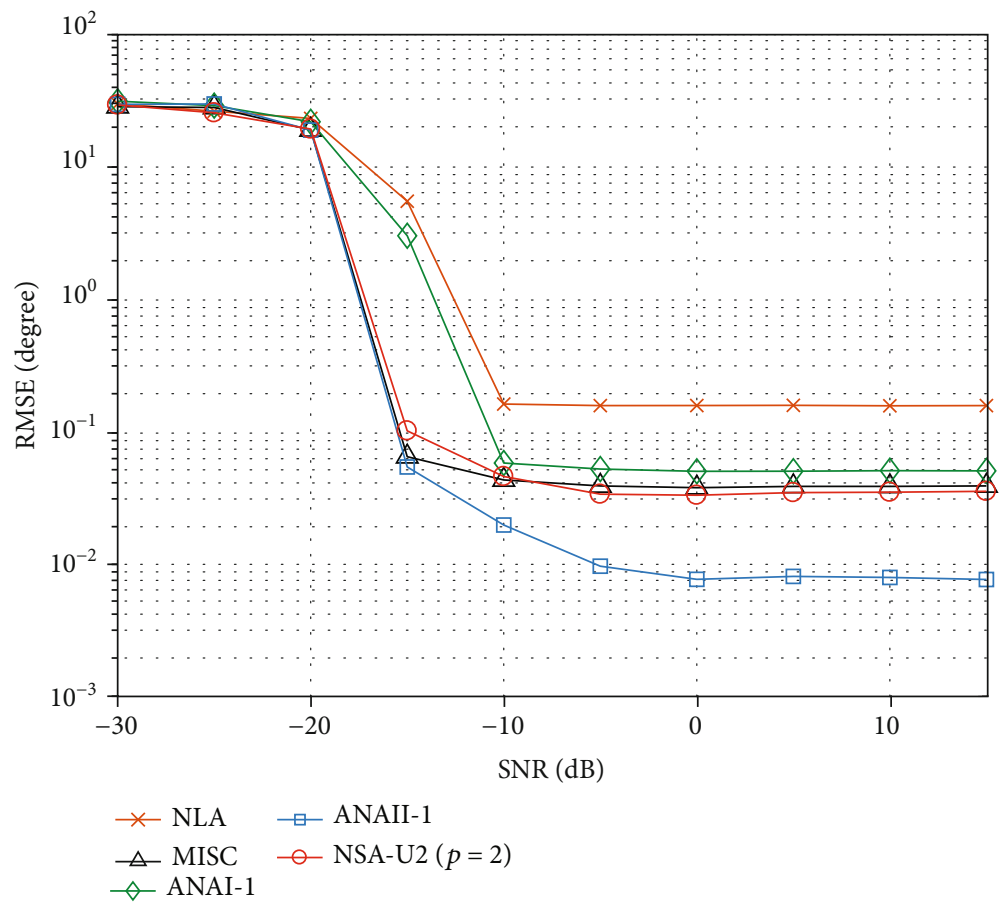

(b)

Figure 6: (a) RMSE of NSA-U2 under different $p$ versus SNR (b) RMSE of different sparse arrays versus SNR.

\section{Simulation Results}

The definition of root mean square error (RMSE) is given as follows,

$$
\mathrm{RMSE}=\sqrt{\frac{1}{Q K} \sum_{q=1}^{Q} \sum_{k=1}^{K}\left(\theta \wedge_{k, q}-\theta_{k}\right)^{2}}
$$

$Q$ is the number of Monte Carlo, $K$ is the number of target signals, and $\widehat{\theta}_{k, q}$ is the DOA of the $k$ th estimated source by the $q$ th Monte Carlo experiment.

Simulation 9. The MUSIC spectrum in NSA-U2 under different $p$.

When we use DAA [?] to estimate DOAs, the maximum number of detectable signals is $\left(S_{v}-1\right) / 2$ in theory, which is 


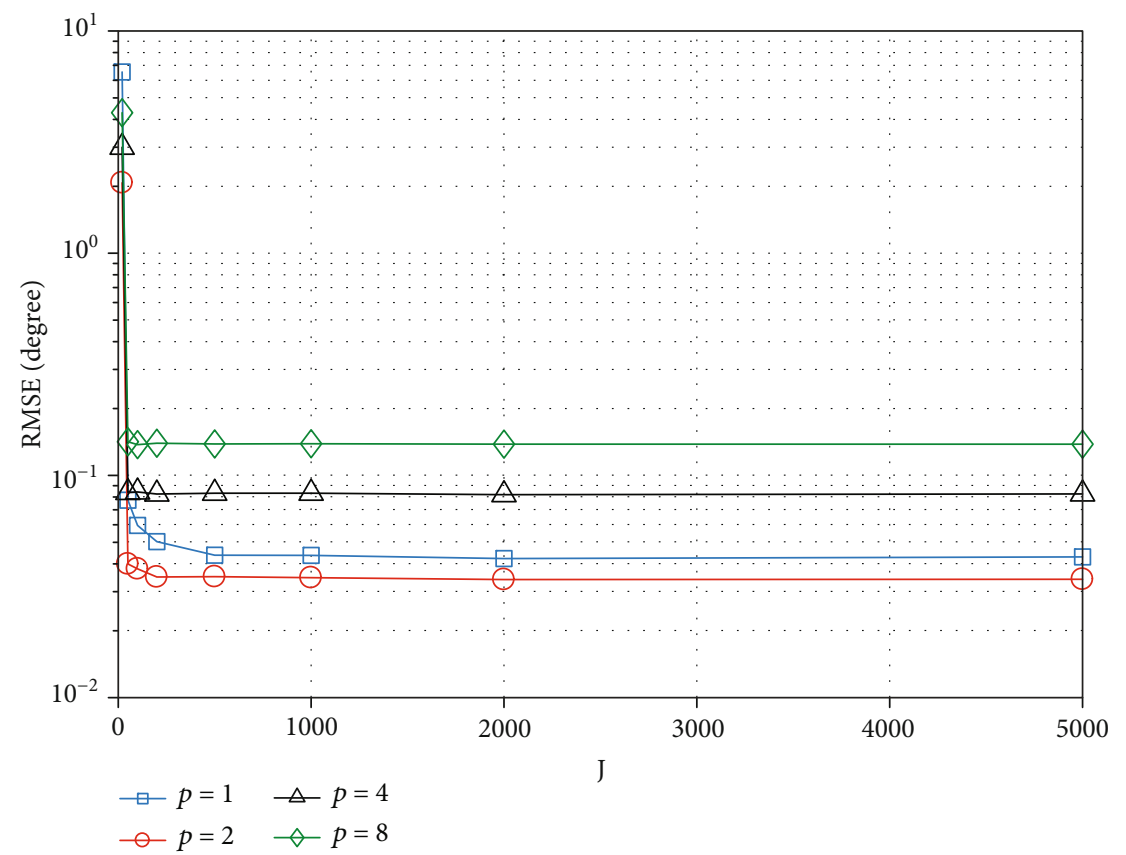

(a)

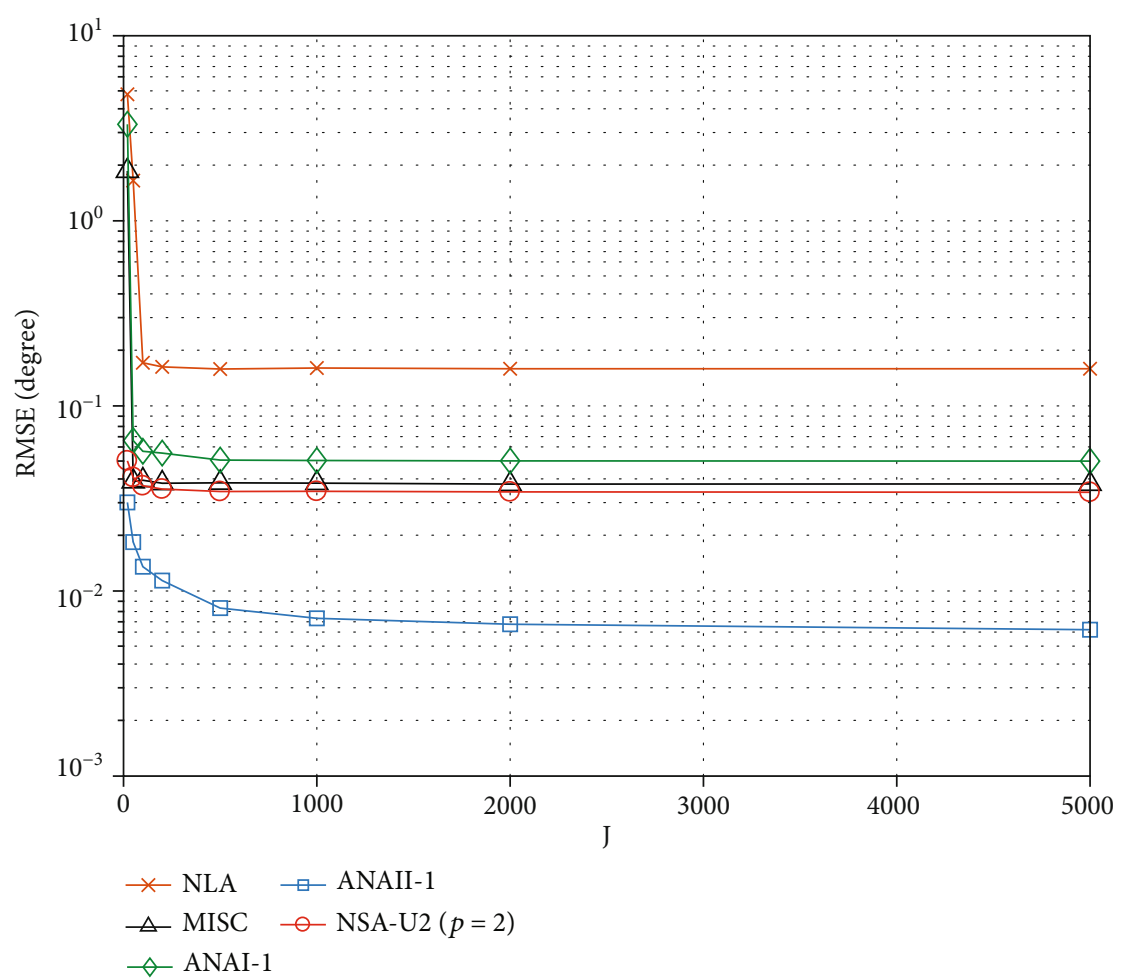

(b)

FiguRe 7: (a) RMSE of NSA-U2 under different $p$ versus snapshot (b) RMSE of different sparse arrays versus snapshots.

much more than $T$. In first simulation, we present the MUSIC spectrum with mutual coupling, when there are multiple signals more than $T$. Assume $K=25$ located at $\theta_{k}=0^{\circ}$ $+\Delta \theta(K / 2+1 / 2-k), 1 \leq k \leq 25$ and $\mathrm{SNR}=10 \mathrm{~dB}, J=5000$. Set $T=16, M=8, N=9$, and $\Delta \theta=3.75^{\circ}$ and $p$ can be $\{1,2$, $4,8\}$. Moreover, set $B=3, c_{1}=0.3 e^{j \pi / 3}$, and $c_{b}=c_{1} e^{-j \pi(b-1) / 8}$.
Thus, we apply DAA and obtain the spectrum under different $p$, which is shown in Figure 4. The figure shows that we can estimate underdetermined signals in NSA-U2. However, with the increase of $p$, the influence of mutual coupling to DOA estimation is more obvious, where the peaks (blue lines) gradually deviate from the true DOAs (red lines). 


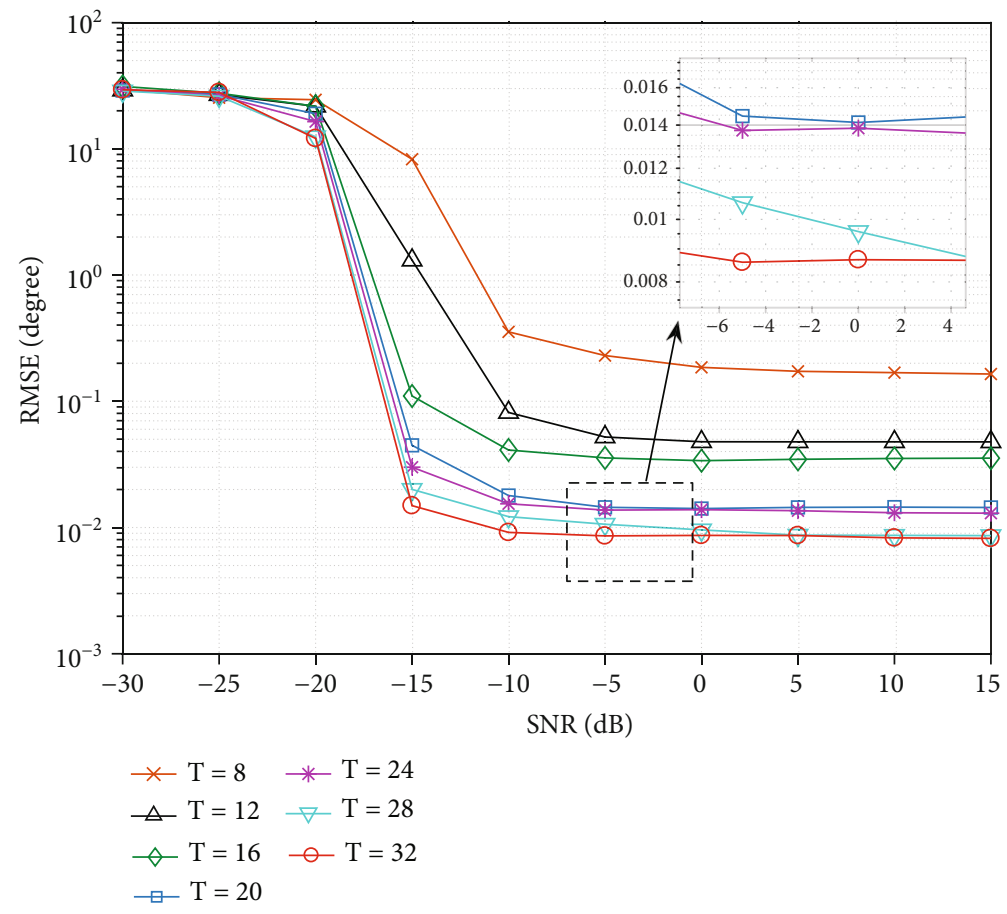

Figure 8: The RMSEs of NSA-U2 under different $T$.

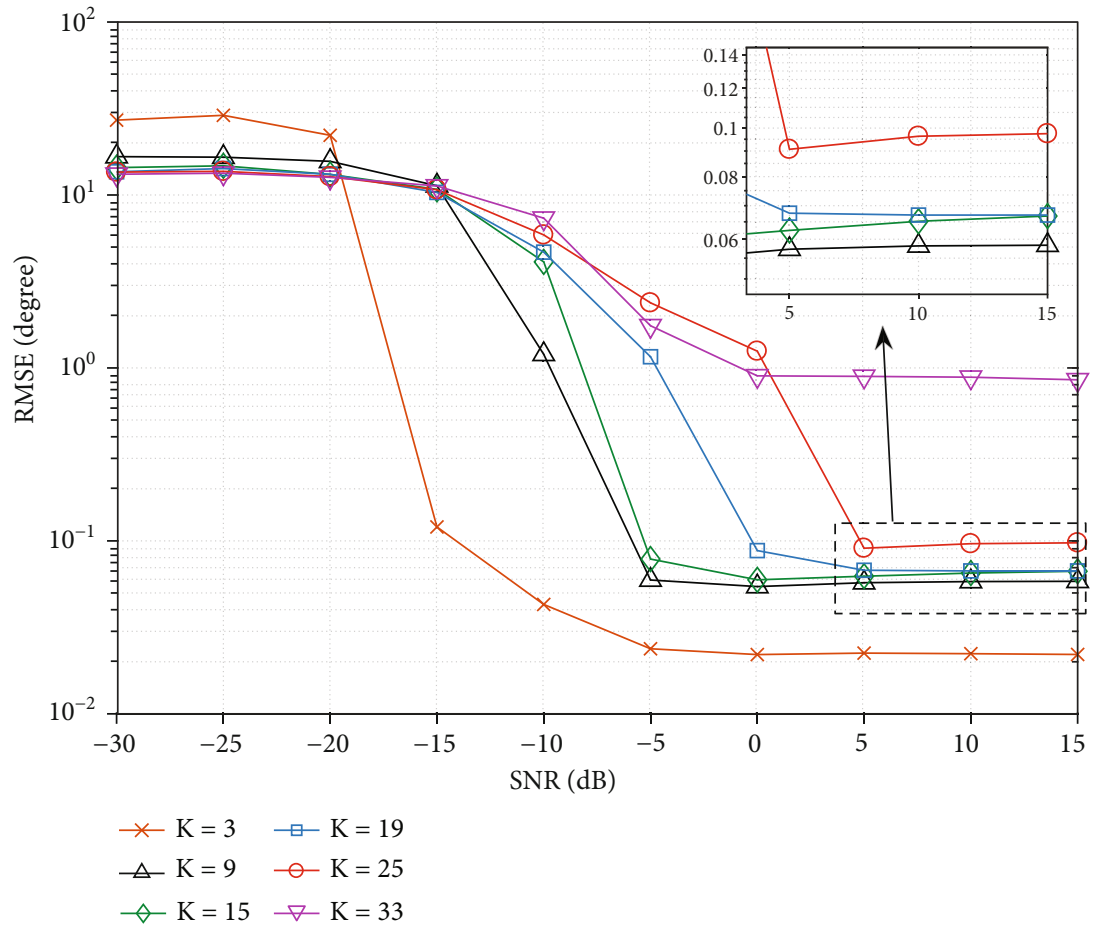

FIGURE 9: The RMSEs of NSA-U2 under different $K$.

Moreover, we set $p=2$ and vary the $\Delta \theta$ from $\left\{5^{\circ}, 6.67^{\circ}\right.$, $\left.2.5^{\circ}, 1.67^{\circ}\right\}$. The spectrum is shown in Figure 5. When the target angle difference increases, if the DOAs of signals are more close to $90^{\circ}$, the accuracy will decrease or even deterio- rate. The RMSE of $\Delta \theta=5^{\circ}$ is $0.087^{\circ}$, while the RMSE of $\Delta \theta$ $=3.75^{\circ}$ is $0.071^{\circ}$. And when $\Delta \theta=6.67^{\circ}$, the DOA estimation of signal from the direction higher than $60^{\circ}$ fails. When the target angle difference decreases, it can have the higher 


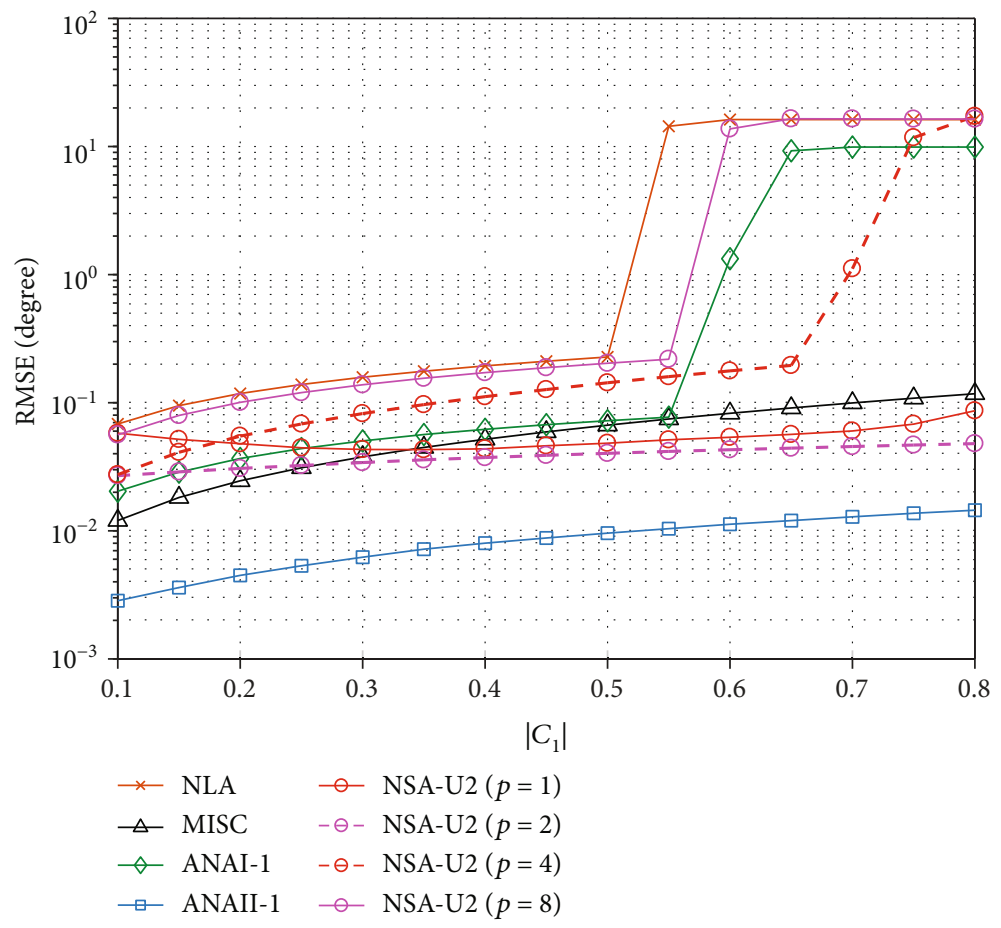

Figure 10: The RMSEs of different sparse arrays under different $\left|c_{1}\right|$.

accuracy, such as $\Delta \theta=2.5^{\circ}$, whose RMSE is $0.04^{\circ}$. But if the difference is lower than the resolution of NSA-U2, such as $\Delta \theta=1.67^{\circ}$, the DOA estimation fails.

Simulation 10. RMSE performance comparison of different SNRs.

In this simulation, we show the RMSE performance with mutual coupling under different SNRs. The mutual coupling coefficients and the structure of NSA-U2 are the same as simulation 9, and SNR is from $-30 \mathrm{~dB}$ to $15 \mathrm{~dB}$ with $5 \mathrm{~dB}$ intervals. Set $Q=500$, and results are shown in Figure 6(a). When SNR $>-20 \mathrm{~dB}$, the RMSEs decrease with SNR increasing, but when SNR $>-5 \mathrm{~dB}$, the RMSEs go to be flat due to the mutual coupling. When $p>2, L e$ is the main factor to affect the RMSEs, but when $p \leq 2, S_{v}$ is the main factor. So, when $p=2$, NSA-U2 can obtain the highest accuracy. Moreover, we compare the NSA-U2 under $p=2$, with NLA, MISC, ANAI-1, and ANAII-1, where the RMSEs are shown in Figure 6(b). Considering Figure 3, this figure shows that the RMSE of NSA-U2 is lower than NLA, ANAI-1, and MISC due to the weaker mutual coupling. ANAII-1 has the lowest RMSE because it has the largest $S_{v}$, but the complexity to design ANAII-1 is much higher than NSA-U2, especially when $T$ is big.

Simulation 11. RMSE performance comparison of different snapshots.

Similar to the simulation 10 , we set $\mathrm{SNR}=10 \mathrm{~dB}$ and vary $J$ from $\{50,100,200,500,1000,2000,5000\}$. The RMSEs are shown in Figure 7 . The simulation results show that the performance of RMSEs is improved with the increase of snapshot numbers, but when $J>500$, RMSEs are gradually flatted. Other conclusions are the same as that in simulation 10 .

Simulation 12. RMSE performance comparison of different $T$

Based on the setting of simulation 10, we further consider the RMSEs of NSA-U2 with different numbers of sensors defined as $T$ under $p=2$. We vary $T$ from $\{8,12$, $16,20,24,28,32\}$. Through the conclusion in Table 2, we can have the corresponding values of $S_{v}$ as $\{33,75,115,1$ $85,245,343,423\}$. The results are shown in Figure 8 . The figure shows that the bigger $T$ can cause the bigger $S_{v}$, which means the higher accuracy. But due to the mutual coupling, the RMSEs goes to be flat when SNR $>-5 \mathrm{~dB}$, and the gap between the RMSEs corresponding to the bigger $T$ is not obvious. Although the difference of $S_{v}$ between $T=28$ and $T=32$ and that between $T=20$ and $T=24$ is big, the RMSEs between $T=28$ and $T=32$ and that between $T=20$ and $T$ $=24$ are very close.

Simulation 13. RMSE performance comparison of different $K$

In this simulation, we do the experiments about the effect of number of signals to the RMSEs. Based on the setting of simulation 10 , we set $\theta_{k}=0^{\circ}+\Delta \theta(K / 2+1 / 2-k), 1 \leq k \leq K$, $\Delta \theta=3.75^{\circ}$, and vary $K$ from $\{3,9,15,19,25,33\}$. The results in Figure 9 show that when SNR $>-5 \mathrm{~dB}$, with the increase of $K$, the RMSEs increase. If $K$ is small, such as $K=3$, the threshold, defined as the value of SNR when $\mathrm{RMSE}=1^{\circ}$, is even smaller than $-15 \mathrm{~dB}$. The threshold becomes bigger with $K$ increasing. Moreover, the RMSEs of $K=25$ and $K=33$ 
also prove that the feasibility of estimating underdetermined signals under enough SNR, where it needs SNR $\geq 0 \mathrm{~dB}$ shown in figure.

Simulation 14. RMSE performance comparison of different $\left|c_{1}\right|$.

$\left|c_{1}\right|$ determines the strength of mutual coupling. So, in this simulation, we compare the RMSEs of different sparse arrays under different $\left|c_{1}\right|$. The sparse arrays are the same as simulation 10 . We set $\mathrm{SNR}=10 \mathrm{~dB}$ and $J=5000 .\left|c_{1}\right|$ varies from 0.1 to 0.8 with 0.05 intervals. The RMSEs are shown in Figure 10. For all array geometries, as $\left|c_{1}\right|$ increases, which causes more severe mutual coupling effect, the associated RMSEs increase. And NSA-U2 under $p=1$ and $p=2$ has the similar robust DOA estimation as MISC and ANAII-1 facing severe mutual coupling.

\section{Conclusion}

In this paper, based on GCLA, the design of sparse array using two ULAs is proposed, named NSA-U2. The paper analyzes the relationship between the displacement $L$ between two subarrays and the number of consecutive lags $S_{v}$ in difference coarray. Then, the selection of the number of subarray sensors and the analytical expressions of $L, S_{v}$ are derived, which can obtain the maximum number of consecutive lags. At last, facing mutual coupling, the paper analyzes the balance between $S_{v}$ and Le in NSA-U2. Through the simulations, NSA-U2 both has a low-complexity design method and obtains the robust DOA estimation facing severe mutual coupling under proper $p$. Considering the advantages of NSA-U2, the design of sparse array using $P$ ULAs can be conducted in the future research.

\section{Appendix}

\section{A. Proof of Theorem 3}

Lemma 15. If there exists a positive value $-\bar{\ell}_{c}$, where both $\bar{\ell}_{c} \in \mathbb{D}_{h n}$ and $\bar{\ell}_{c} \in \mathbb{D}_{c}$, then any positive hole, where $\ell_{h} \in \mathbb{D}_{h n}$ and $\ell_{h} \leq-\bar{\ell}_{c}$, can have $\ell_{h}=-\ell_{c}$, where $-\ell_{c} \in \mathbb{D}_{c}^{-}$.

Proof. Let $\bar{\ell}_{h}=-\bar{\ell}_{c}$. Due to the property (b) of Proposition 2, other positive holes are located at $\mathbb{D}_{h n}^{\prime}=\left\{\ell_{h}^{\prime} \mid \ell_{h}^{\prime}=\bar{\ell}_{h}-(a\right.$ $\bar{M}+b N)\}$, where $a \geq 0, b \geq 0$. Then, considering the property (a) of Proposition 2, other negative lags of $\mathbb{D}_{c}$, which are no less than $\bar{\ell}_{c}$, are located at $\mathbb{D}_{c n}^{\prime}=\left\{\ell_{c}^{\prime} \mid \ell^{\prime}{ }_{c}=\bar{\ell}_{c}+\left(a^{\prime} \bar{M}+b^{\prime} N\right.\right.$ )$\}$, where $a^{\prime} \geq 0, b^{\prime} \geq 0$. When $-\bar{\ell}_{c}=\bar{\ell}_{h}$, we have known that $a, a^{\prime}$ and $b, b^{\prime}$ have the same range of values; so, $\mathbb{D}_{h n}^{\prime}=-\mathbb{D}$ ${ }_{c n}$. Due to $-\mathbb{D}^{\prime}{ }_{c n} \subset \mathbb{D}_{c}^{-}$, the lemma is true.

lemma 15 reveals that all positive holes in $\mathbb{D}_{h n}$ can be aligned by $\mathbb{D}_{c}^{-}$, when $-\bar{\ell}_{c}=(\bar{M}-1)(N-1)+L-1$. Therefore, let $\bar{\ell}_{c}$ $=L+a_{1} \bar{M}+a_{2} N$, where $a_{1} \geq 0, a_{2} \geq 0$, and

$$
-\left(L+a_{1} \bar{M}+a_{2} N\right)=(\bar{M}-1)(N-1)+L-1,
$$

which can be simplified to

$$
L=-\frac{1}{2}\left(\bar{M} N-\bar{M}-N+a_{1} \bar{M}+a_{2} N\right)
$$

Then, $S_{c}$ can be $2\left(M^{\prime} N+L-1\right)+1$. (A.2) requires that $L$ should be divisible by 2 and as big as possible. The former requirement is depended on the odevity of $\bar{M}, N$ and values of $a_{1}, a_{2}$. The latter requirement means that $a_{1}=0$ orl and $a_{2}=0$ or 1 . When $a_{1}=a_{2}=0, L$ can never be divided by 2 ; so, $a_{1}, a_{2}$ cannot be 0 simultaneously.

Moreover, we find that some values of $L$ let two subarrays have one common sensor, where $L$ meets that

$$
N m+\bar{M}(N-1)+L=\bar{M} n .
$$

The possible values of some parameters are listed in Table 3. Thus, with a fixed value of $T$, the number of sensors of subarray 2 can be $M$; so, $S_{c}$ can be bigger as $2(M N+L)-1$.

\section{B. Proof of Theorem 4}

Situation 16. We first consider the situation that $S_{c}$ reaches the maximum values, and then all values in $\mathbb{D}_{h n}$ can be aligned. Based on theorem 3, the number of consecutive lags in $\mathbb{D}_{c} \cup \mathbb{D}_{c}^{-}$is $S_{c}=2(M N+L)-1$. Next, we expect that the minimum value in $\mathbb{D}_{h p}$ can be aligned by $\mathbb{D}_{s}$; so, we have

$$
\begin{gathered}
M N-1+L+1=N m, \\
M N-1+L+1=\bar{M} n .
\end{gathered}
$$

(B.1) and (B.2), respectively, tell that $L$ should be divisible by $N$ and $\bar{M}$.

Case 17. If $L$ meets (B.1), the next hole is located at $M N+L$ $+\bar{M}$, which cannot be divisible by $N$. So, $S_{v}$ is given by $S_{v}$ $=S_{c}+2 \bar{M}$.

Case 18. In order to let $L$ meet (B.2), $\bar{M} n$ should be no less than $M N+L$. When $p>1, M N+L>\bar{M}(N-1)$ for any value of $L$. Thus, if $p=1$ and $L$ meets (A.3), the hole located at $M$ $N+L+N$ cannot be divisible by $\bar{M}$; so, $S_{v}=S_{c}+2 N$.

If $L=(\bar{M} N-\bar{M}) / 2$, which can be divisible by $\bar{M}, S_{v}=S_{c}+2$ $N=2 M N-\bar{M} N+2 N+\bar{M}-1$. If $L=(\bar{M} N-N) / 2$, which can be divisible by $N, S_{v}=S_{c}+2 \bar{M}=2 M N-\bar{M} N+N+2 \bar{M}$ - 1. And If $L=\bar{M} N / 2$, which can be divisible by both $\bar{M}$ and $N$, both $S_{v}=2 M N-\bar{M} N+2 N-1$ and $S_{v}=2 M N-\bar{M}$ $N+2 \bar{M}-1$. Hence, $S_{v}=2 M N-\bar{M} N+2 N+\bar{M}-1$ is the biggest, $S_{v}=2 M N-\bar{M} N+N+2 \bar{M}-1$ and $S_{v}=2 M N-\bar{M}$ $N+2 N-1$ that which one is bigger is depended on $N-2$ $\bar{M}$ are positive or not, and $S_{v}=2 M N-\bar{M} N+2 \bar{M}-1$ are the smallest. In order to maximize value of $S_{v}$, we need to let $L$ be as large as possible and also meet (B.1) or (B.2). Thus, we need to discuss odevity of parameters to make sure the values of $S_{v}$.

We first consider $p=1$. If $N$ is odd and no matter $\bar{M}$ is even or odd, $L=-(\bar{M} N-\bar{M}) / 2$ can be divisible by 2 , and $S_{v}$ $=M N+M+2 N-1$. If $N$ is even and $\bar{M}$ is odd, $L=-(\bar{M} N$ 
$-N) / 2$ and $L=-\bar{M} N / 2$ can be divisible by 2 , then if $N<2 \bar{M}$, $S_{v}=M N+N+2 M-1$; otherwise, $S_{v}=M N+2 N-1$. And we then consider $p>1$. If $\bar{M}$ is odd and no matter $N$ is even or odd, $L=-(\bar{M} N-N) / 2$ can be divisible by 2 , and $S_{v}=2$ $M N-\bar{M} N+N+2 \bar{M}-1$. If $\bar{M}$ is even and $N$ is odd, $L=-$ $\bar{M} N / 2$ can be divisible by 2 , and $S_{v}=2 M N-\bar{M} N+2 \bar{M}-1$. At last, for the sake of clarity, we list the value of $L$ and the analytical expression of $S_{v}$ in Table 2 under different situations.

Situation 19. In this part, we consider the situation that the hole $(\bar{M}-1)(N-1)+L-1$ cannot be aligned by $\mathbb{D}_{c}^{-}$; so, it must be aligned by $\mathbb{D}_{s}$. Assume that $\bar{\ell}_{c}=(\bar{M}-1)(N-1)+L$ $-1-a_{1} M-a_{2} N$, where $a_{1} \geq 0, a_{2} \geq 0$. If $(\bar{M}-1)(N-1)+$ $L-1=M n$, then $a_{1}=0, a_{2}=1$; otherwise, if $(\bar{M}-1)(N-1)$ $+L-1=N m$, then $a_{1}=1, a_{2}=0$. Considering that $L$ should be as big as possible, we set $-L=\bar{\ell}_{c}$; so,

$$
L=-\frac{1}{2}\left(\bar{M} N-\bar{M}-N-a_{1} \bar{M}-a_{2} N\right) .
$$

In order to ensure whether there is a common sensor in this situation, take (B.3) into (A.3), and each side is divided by $\bar{M}$, which can be expressed as

$$
\begin{aligned}
\frac{N}{\bar{M}} & m+N-1-\frac{1}{2}\left(N-1-a_{1}-\frac{N+a_{2} N}{\bar{M}}\right) \\
& =n \longrightarrow \frac{N}{\bar{M}}\left(m+\frac{1+a_{2}}{2}\right) \\
& =n+\frac{1-a_{1}-N}{2} .
\end{aligned}
$$

So, $m+\left(1+a_{2}\right) / 2=\bar{M}$ and $n+\left(1-a_{1}-N\right) / 2=N$, but $n+\left(1-a_{1}-N\right) / 2<N$; thus, (B.4) is false, and two subarrays have no common sensors. Then, we make sure whether the minimum value of $\mathbb{D}_{h p}$ given by $M^{\prime} N+L$ can be aligned by $\mathbb{D}_{s}$, so we have

$$
M N-N+L=N \operatorname{mor} M N-N+L=M n .
$$

Take (B.3) into (B.5), and each side of each equation is divided by $N$ and $\bar{M}$, respectively; then,

$$
\begin{aligned}
& M-1-\frac{1}{2}\left(\bar{M}-\frac{\bar{M}}{N}-1-1\right)=\text { mor, } \\
& p N-\frac{N}{\bar{M}}-\frac{1}{2}\left(N-1-\frac{N}{\bar{M}}-1\right)=n .
\end{aligned}
$$

(B.6) indicates that there are no integer values $m$ and $n$ to satisfy (B.5); so, the values in $\mathbb{D}_{h p}$ cannot be aligned by $\mathbb{D}_{s}$. As a result, the maximum number of consecutive lags $S_{v}$ is 2( $\left.M^{\prime} N+L\right)-1$.

Hence, we compare $S_{v}$ that we can obtain in two situations. In situation 19, the maximum values of $S_{v}$ can be $2 M$ $N-\bar{M} N+\bar{M}-1$, when $L=(\bar{M} N-\bar{M}-N-N)$. Thus, when $p=1, S_{v}=M N+M-1$ is smaller than any case in situation 16. And when $p>1, S_{v}=2 M N-\bar{M} N+\bar{M}-1$ is also smaller than any case in situation 16 . So, we can obtain the maximum number of consecutive lags in situation 16, and the theorem is proved.

\section{Proof of Theorem 5}

Considering that $S_{v}$ is decided by $\bar{M}, N$, we define a function

$$
f(N)=2 M N-\frac{M N}{p}+a \frac{M}{p}+b N-1
$$

where $M=T-N+1,1 \leq p \leq T$, and $a, b$ are integers chose from $\{0,1,2\}$. Then, take the derivative of the function and have

$$
f^{\prime}(N)=\frac{d f}{d N}=2 T-4 N+\frac{2 N}{p}+b+2-\frac{T+a+1}{p} .
$$

Let $f^{\prime}(N)=0$ and have $N=(2 T p+2 p+b p-T-a-1) /$ $(4 p-2)$. Thus, define a new function about $p$ as $g(p)=(2 T$ $p+2 p+b p-T-a-1) /(4 p-2)$. Take the derivative of it and have $g^{\prime}(p)=d g / d p=(8+4 a-2 b) /(4 p-2)^{2}$, where $g^{\prime}($ $p) \geq 0$ for every $p, a, b$. Thus, we only discuss the situations that $p=1$ and $p=T$. Based on the conclusions in Table 2, we give the detailed analysis.

Case 19. When $p=1, g(p)=(T+1+b-a) / 2$. If $M$ is even and $N$ is odd, $T$ is even, and $a=1, b=2$ and $N=T / 2+1, M$ $=T / 2$. If $M$ is odd and $N$ is even, due to $N \leq 2 M, T$ is even, and $a=2, b=1$ and $M=T / 2+1, N=T / 2$. Those two situations are same. Because we assume $N>M$, when $T$ is even, $N=T / 2+1, M=T / 2$. If $M$ is odd and $\mathrm{N}$ is odd, $T$ is odd, and $a=1, b=2$ and $N=T / 2+1 / 2, M=T / 2+1 / 2$. Because $M, N$ are coprime integers, when $T$ is odd, and $N=T / 2+3$ $/ 2, M=T / 2-1 / 2$.

Case 20. When $p=T, a=2$ and $g(p)=\left(2 T^{2}+T+b T-2\right) /($ $4 T-2)$. If $M$ is even and $N$ is odd, $T$ is even, $b=0$, and $N$ $=\left(2 T^{2}+T-2\right) /(4 T-2)<T / 2+1 / 2$. So, $N=T / 2+1$ and $M=T / 2$. If $M$ is odd and $N$ is even, $T$ is even, $b=1$, and $N$ $=\left(2 T^{2}+2 T-2\right) /(4 T-2)<T / 2+1 / 2$. So, $N=T / 2+1$ and $M=T / 2$. If $M$ is odd and $N$ is odd, $T$ is odd, and $b=1$ and $N=T / 2+1 / 2, M=T / 2+1 / 2$. Because $M, N$ are coprime integers, when $T$ is odd, $N=T / 2+3 / 2, M=T / 2-1 / 2$.

In conclusion, when $T$ is even, $N=T / 2+1, M=T / 2$ and when $T$ is odd, $N=T / 2+3 / 2, M=T / 2-1 / 2$.

When $M, N$ are the fixed integers, we consider the function of $p$, where

$$
f(p)=2 M N-\frac{M N}{p}+a \frac{M}{p}+b N-1 .
$$


Take the derivative of it and have

$$
f^{\prime}(p)=\frac{d f}{d p}=\frac{(N-a) M}{p^{2}} .
$$

Obviously, $f^{\prime}(p)>0$ for every $p$, which means with the increase of $p, S_{v}$ increases.

\section{Proof of Proposition 8}

(a) If we assume that there exists another pair $\left(m^{\prime}, n^{\prime}\right)$ meeting that

$$
\bar{M}(N-1)+L+N m-M n=\bar{M}(N-1)+L+N m^{\prime}-M n^{\prime},
$$

which can be rewritten as

$$
N\left(m-m^{\prime}\right)=\bar{M}\left(n-n^{\prime}\right)
$$

Because $\bar{M}, N$ are coprime integers and $-N+1 \leq n-n^{\prime}$ $\leq N-1$, then $m-m^{\prime}=n-n^{\prime}=0$ and $m=m^{\prime}, n=n^{\prime}$. Thus, the assumption is false. And each element in $\mathbb{D}_{c}$ only corresponds to one unique $(m, n)$; so, $w(\ell)=1, \ell \in \mathbb{D}_{c}$.

(b) Due to $\mathbb{D}_{c}^{-}=-\mathbb{D}_{c}$, it also satisfies the property (a). Based on theorem $3, \mathbb{D}_{c}^{-}$and $\mathbb{D}_{c n}$ have no common part. However, $\mathbb{D}_{c}^{-}$and $\mathbb{D}_{c c}$ have one common part $\left\{\ell \mid \ell=\bar{\ell}_{c}+a \bar{M}+b N\right\}$, where $a \geq 0, b \geq 0$. And due to $\bar{\ell}_{c} \in \mathbb{D}_{c n}, a, b$ cannot be 0 at the same time. Because the maximum value of $\mathbb{D}_{c}^{-}$is $-L$, every $\ell$ in the common part has $\ell \leq-L$. Hence, considering property (a), the value of weight function of the common part is 2. Moreover, the two subarrays have one common sensor; so, $w(0)=2$.

$\mathbb{D}_{s} \cup \mathbb{D}_{s}^{-}$is the self-coarray lags; so, the weight function of each uniform linear subarray is $w(0)=T$, and $w( \pm \bar{M})$ $=N-1, w(N)=\bar{M}-1$.

(d) Because $\mathbb{D}_{v}=\mathbb{D}_{c} \cup \mathbb{D}_{c}^{-} \cup \mathbb{D}_{s} \cup \mathbb{D}_{s}^{-}$, the value of weight function of $\mathbb{D}_{v}$ is the sum of that of the weight function of each subset.

\section{Data Availability}

The authors claim that the data used in this article are provided by their simulations, and this is developed without using any data in a published article to support their results. The data used to support the findings of this study are available from the corresponding author upon request.

\section{Conflicts of Interest}

The authors declare that they have no conflicts of interest.

\section{Acknowledgments}

The authors claim that the data used in this article are provided by our simulations.

\section{References}

[1] F. Sun, Q. Wu, P. Lan, G. Ding, and L. Chen, "Real-valued DOA estimation with unknown number of sources via reweighted nuclear norm minimization," Signal Processing, vol. 148, pp. 48-55, 2018.

[2] F. Sun, P. Lan, and G. Zhang, "Reduced dimension based twodimensional DOA estimation with full DOFs for generalized co-prime planar arrays," Sensors, vol. 18, no. 6, 2018.

[3] A. Moffet, "Minimum-redundancy linear arrays," IEEE Transactions on Antennas and Propagation, vol. 16, no. 2, pp. 172175, 1968.

[4] P. P. Vaidyanathan and P. Pal, "Sparse sensing with coprime samplers and arrays," IEEE Transactions on Signal Processing, vol. 59, no. 2, 2011.

[5] P. Pal and P. P. Vaidyanathan, "A novel array structure for directions-of-arrival estimation with increased degrees of freedom," in 2010 IEEE International Conference on Acoustics, Speech and Signal Processing, Dallas, TX, USA, 2010.

[6] P. Pal and P. P. Vaidyanathan, "Nested arrays: a novel approach to array processing with enhanced degrees of freedom," IEEE Transactions on Signal Processing, vol. 58, no. 8, pp. 4167-4181, 2010.

[7] P. Pal and P. P. Vaidyanathan, "Coprime sampling and the music algorithm," Digital Signal Processing Workshop \& IEEE Signal Processing Education Workshop, 2011.

[8] C. L. Liu and P. P. Vaidyanathan, "Remarks on the spatial smoothing step in coarray music," IEEE Signal Processing Letters, vol. 22, no. 9, pp. 1438-1442, 2015.

[9] M. Wang and A. Nehorai, "Coarrays, music, and the cramrrao bound," IEEE Transactions on Signal Processing, vol. 65, no. 4, pp. 933-946, 2016.

[10] C. L. Liu and P. P. Vaidyanathan, "Comparison of sparse arrays from viewpoint of coarray stability and robustness," 2018 IEEE 10th Sensor Array and Multichannel Signal Processing Workshop (SAM), 2018.

[11] C. L. Liu and P. P. Vaidyanathan, "Robustness of difference coarrays of sparse arrays to sensor Failures-Part I: A Theory Motivated by Coarray MUSIC," IEEE Transactions on Signal Processing, vol. 67, no. 12, pp. 3213-3226, 2019.

[12] C. L. Liu and P. P. Vaidyanathan, "Robustness of difference coarrays of sparse arrays to sensor Failures-Part ii: Array geometries," IEEE Transactions on Signal Processing, vol. 67, no. 12, pp. 3227-3242, 2019.

[13] C.-L. Liu and P. Vaidyanathan, "Novel algorithms for analyzing the robustness of difference coarrays to sensor failures," Signal Processing, vol. 171, p. 107517, 2020.

[14] Y. D. Zhang, S. Qin, and M. G. Amin, "Doa estimation exploiting coprime arrays with sparse sensor spacing," in 2014 IEEE International Conference on Acoustics, Speech and Signal Processing (ICASSP), Florence, Italy, 2014.

[15] S. Qin, Y. D. Zhang, and M. G. Amin, "Generalized coprime array configurations for direction-of-arrival estimation," IEEE Transactions on Signal Processing, vol. 63, no. 6, pp. 13771390, 2015. 
[16] S. Ren, W. Wang, and Z. Chen, "Doa estimation exploiting unified coprime array with multi-period subarrays," in 2016 CIE International Conference on Radar (RADAR), Guangzhou, China, 2016.

[17] S. M. Alamoudi, M. A. Aldhaheri, S. A. Alawsh, and A. H. Muqaibel, "Sparse doa estimation based on a shifted coprime array configuration," in 2016 16th Mediterranean Microwave Symposium (MMS), Abu Dhabi, United Arab Emirates, 2017.

[18] C. Liu and P. P. Vaidyanathan, "Super nested arrays: linear sparse arrays with reduced mutual coupling-part i: fundamentals," IEEE Transactions on Signal Processing, vol. 64, no. 15, pp. 3997-4012, 2016.

[19] C. L. Liu and P. P. Vaidyanathan, "Super nested arrays: Linear sparse arrays with reduced mutual coupling - part ii: Highorder extensions," IEEE Transactions on Signal Processing, vol. 64, no. 15, 2016.

[20] J. Liu, Y. Zhang, Y. Lu, S. Ren, and S. Cao, “Augmented nested arrays with enhanced dof and reduced mutual coupling," IEEE Transactions on Signal Processing, vol. 65, no. 21, pp. 55495563, 2017.

[21] Z. Zheng, W. Q. Wang, Y. Kong, and Y. D. Zhang, "MISC array: a new sparse array design achieving increased degrees of freedom and reduced mutual coupling effect," IEEE Transactions on Signal Processing, vol. 67, no. 7, pp. 1728-1741, 2019.

[22] B. Friedlander and A. J. Weiss, "Direction finding in the presence of mutual coupling," IEEE Transactions on Antennas \& Propagation, vol. 39, no. 3, pp. 273-284, 1991.

[23] T. Svantesson, "Modeling and estimation of mutual coupling in a uniform linear array of dipoles," in 1999 IEEE International Conference on Acoustics, Speech, and Signal Processing. Proceedings. ICASSP99 (Cat. No. 99CH36258), Phoenix, AZ, USA, 1999.

[24] Z. Ye, J. Dai, X. Xu, and X. Wu, "Doa estimation for uniform linear array with mutual coupling," IEEE Transactions on Aerospace \& Electronic Systems, vol. 45, no. 1, pp. 280-288, 2009.

[25] J. Dai, W. Xu, and D. Zhao, "Real-valued doa estimation for uniform linear array with unknown mutual coupling," Signal Processing, vol. 92, no. 9, article 20562065, 2012.

[26] B. Liao, Z. G. Zhang, and S. C. Chan, "DOA estimation and tracking of ulas with mutual coupling," IEEE Transactions on Aerospace \& Electronic Systems, vol. 48, no. 1, pp. 891-905, 2012.

[27] U. V. Dias and S. Srirangarajan, "Co-prime arrays and difference set analysis," in 2017 25th European Signal Processing Conference (EUSIPCO), Kos, Greece, 2017. 